\title{
Violencia institucional y obstétrica, algunos indicadores para
} su explicación

\author{
Jorge Carreño-Meléndez* y Claudia Sánchez-Bravo \\ Instituto Nacional de Perinatología, Ciudad de México, México
}

\section{Resumen}

El objetivo de este trabajo es contextualizar la violencia institucional y la obstétrica en la búsqueda de algunos indicadores para su explicación, dado que con frecuencia frente a cualquier acto médico que se tipifica como violento se hace responsable al médico tratante. Se pidió el apoyo de un especialista en ginecología y obstetricia para caracterizar los eventos y los procedimientos médicos que con mayor frecuencia son cuestionados durante la atención del parto y tipificar en esos actos médicos la violencia obstétrica. Se aislaron dieciséis eventos y se confrontó con el procedimiento médico desde la visión de la técnica. Como resultado del análisis, y dada la naturaleza del acto médico, la violencia institucional y la obstétrica puede ser dependientes. Un alto porcentaje de los embarazos que se atienden son de alto riesgo y los especialistas pueden cometer impericia, sin que necesariamente se trate de violencia obstétrica, que vía la capacitación se pueden solucionar. Se observa la importancia en la vida diaria y profesional de la ética con una visión laica para establecer la relación médico-paciente.

Palabras clave: Violencia institucional. Ética médica. Salud en la mujer. Política en salud.

\section{Institutional and obstetrical violence, some markers for its explanation}

\section{Abstract}

The goal of this research is contextualizing institutional and obstetrical violence to look for markers that explain its occurrence, since it usually is classified as violence when compared to any other medical activity while the treating physician is held responsible. Support was asked from a physician specialized in gynecology and obstetrics to characterize the events and medical procedures that are mostly put into question during labor and that help classify these medical activities as obstetrically violent. Sixteen events were selected, isolated and compared to their medical procedures from a technical perspective. The result of the analysis, due to the nature of the medical activity, is that both institutional and obstetrical violence are dependent. A high percentage of treated pregnancies are considered as high-risk pregnancies and specialists may commit negligence that may not be necessarily considered as obstetrical violence and that may be solved with training. The importance of secular ethics in both personal and professional lives to establish the relationship between physician and patient is also noted.

Key words: Institutional violence. Medical ethics. Women's health. Health politics.

\section{Correspondencia:}

*Jorge Carreño-Meléndez

E-mail: Jocame2003@gmail.com
Fecha de recepción: 07-09-2018

Fecha de aceptación: 12-11-2018

DOI: 10.24875/PER.M19000005
Disponible en internet: 25-03-2019 Perinatol Reprod Hum. 2019;33:37-45 www.perinatologia.mx 0187-5337/@ 2018. Instituto Nacional de Perinatología Isidro Espinosa de los Reyes. Publicado por Permanyer México SA de CV. Este es un artículo Open Access bajo la licencia CC BY-NC-ND (http://creativecommons.org/licenses/by-nc-nd/4.0/). 


\section{Introducción}

Tanto en México como en América Latina, gran número de publicaciones recientes hablan sobre la necesidad de retomar los planteamientos desde la ética, lo que se muestra como una tarea urgente. En todos los ámbitos del quehacer humano se habla de los fundamentos éticos que deben estar presentes para el desarrollo de las distintas disciplinas, por eso se tiene que actualizar el debate para encontrar el sentido de la ética en las distintas áreas profesionales y su función en la vida diaria. En la antropología, la sociología, la psicología y en la medicina, entre otras, con sus vertientes, la docencia e investigación están actualizando de manera expedita códigos sobre el comportamiento, aunque muy similares a los manuales de buenas costumbres que abundaban durante el siglo XIX como una práctica cotidiana y solo un número reducido de personas los aplicaban. De manera que no puede existir una ley, reglamento o código de ética que norme la totalidad de las conductas habituales o las emergentes.

Es paradójico que, cuando más evidente es la ruptura del tejido social y las contradicciones manifiestas dentro de la sociedad, más proliferan los códigos de ética. A pesar que el estado de las cosas toma un tinte de inmanejable, especialmente en los países latinoamericanos, es importante intentar explicar cómo se llegó a presentar tal situación.

\section{Desde lo social}

La cultura occidental se configuró a partir del modelo de sociedad griega, que junto con la filosofía formaron una visión sobre el significado de ser mujer y ser hombre, uno de cuyos fundamentos era la marcada inferioridad de la mujer ${ }^{1}$ con respecto al hombre. Ese pensamiento que ha construido a la sociedad occidental a lo largo de varios siglos de existencia no ha variado mucho. El concepto original sobre los géneros solo se ha matizado, pero la esencia aún se mantiene, dando lugar a algunas manifestaciones como variantes de esa postura, y el fundamento de la diferencia entre los géneros no se ha resuelto.

La organización social, tal como la conocemos ahora, se fundó a partir de la división de lo masculino y lo femenino. Con base en esta división se determinaron los arreglos sociales, fundamentados en la sobrevalorización de lo masculino y el poder en oposición a lo femenino. Desde el pensamiento patriarcal se fue construyendo la práctica social, donde se crea el código de normas y valores con la que se convive, y puede ampararse de manera encubierta la violencia hacia la mujer al presentarla como un ser en desventaja.

La violencia en general de forma abierta o soterrada se origina en el ámbito social por las condiciones materiales de vida; los valores sociales predominantes influyen decisivamente en el tipo de prácticas que puede producir dentro de una sociedad. En la actualidad, se sigue manteniendo una cultura de jerarquías donde el estereotipo del poder recae en lo masculino, y el poder media las relaciones sociales, pero con el poder hombres y mujeres se comportan de la misma manera, no existe diferencia entre un estilo femenino y el masculino, esto va determinando un tipo de representaciones sociales donde lo importante es el poder y no los géneros, ya que frente a esto, independientemente del papel de género, la expresión del poder es la misma.

Los gobiernos, como lo consideraba Platón ${ }^{1}$, tienen tres finalidades: satisfacer las necesidades básicas de los ciudadanos, defender el territorio y, por último, administrar la sociedad. Desde que el pensamiento neoliberal entra en escena, la mayoría de los gobiernos latinoamericanos fueron cediendo terreno en las instituciones convirtiendo en una mercancía la educación y la salud, desvirtuando así el sentido social de estos derechos. A instancias de los protogobiernos, como es el Fondo Monetario Internacional y la Organización para la Cooperación y el Desarrollo Económicos², se fue modificando el viejo anhelo de que un mayor número de habitantes de los distintos países tuvieran un acceso a los servicios de salud; estos se vio interrumpido por las veloces privatizaciones y el desmantelamiento de la salud pública, vía la subrogación de los servicios que, por lo general, son otorgados a la familia de los administradores de turno, convirtiéndose en un negocio para las personas que ostentan el poder y dejando a los trabajadores sanitarios excluidos, lo que supone el ejercicio de violencia institucional.

La violencia institucional solo genera impotencia, al ser una práctica cotidiana, porque los individuos al investirse con el poder pueden violar todos los procedimientos ${ }^{3}$ dirigiendo los beneficios solo a sus intereses, casi siempre monetarios, violentando la atención a la paciente ${ }^{4}$, así como la vida académica e institucional.

Al ser el Estado el que tiene que garantizar la convivencia en la vida social, al fallar en su práctica con la impunidad o el abuso, se promueve o se va construyendo la violencia institucional, que es aquella realizada por las/los funcionarias/os, profesionales, personal y 
agentes pertenecientes a cualquier órgano, ente o institución pública ${ }^{5}$.

Otra acepción refiere que la violencia institucional se entiende como aquellos actos $u$ omisiones de las personas en calidad de servidor público que discriminen o tengan como fin dilatar, obstaculizar o impedir el goce y ejercicio de los derechos de las mujeres, así como su acceso al disfrute de las políticas públicas destinadas a prevenir, atender, investigar, sancionar y erradicar los diferentes tipos de violencia ${ }^{5}$.

La expresión del poder en su forma indiferenciada entre los sexos se puede observar en las residencias de las especialidades ${ }^{6}$ y en la investigación. Y es más clara en la consulta diaria con mujeres y hombres investidos con el poder, que dentro de las instituciones de salud se convierten en posibles emisores de violencia; un dato reciente es que en la medida que se está feminizando la especialidad de ginecología y obstetricia aumentan los reportes de violencia obstétrica ${ }^{7}$.

A la violencia institucional se suma a la violencia obstétrica, conceptualizada como todo abuso, acción u omisión intencional, negligente y dolosa que lleven a cabo los profesionistas, técnicos, auxiliares y similares de las diferentes áreas médicas que dañen, denigren, discriminen y brinden un trato inhumano a la mujer durante el embarazo, parto y puerperio; que tengan como consecuencia la pérdida de la autonomía y la capacidad de decidir libremente sobre su cuerpo y la sexualidad ${ }^{5}$.

Bajo estas condicionantes en cualquier momento la paciente puede referir violencia obstétrica durante el proceso de atención al enfrentarse a posibles emisores de violencia.

Dentro de la relación médico-paciente la violencia obstétrica ${ }^{8,9}$ con frecuencia se utiliza como un término común frente a distintas intervenciones médicas a mujeres, sin diferenciar entre violencia institucional y violencia obstétrica por razón de tener un origen distinto. Debido a esto en los últimos quince años un porcentaje importante de médicos tienen un seguro que los protege contra demandas, ya sea los que laboran en una institución o de manera privada, convirtiendo el ejercicio de medicina en una profesión de alto riesgo ${ }^{10}$, lo que provoca la práctica de una medicina defensiva con temores de hasta dónde intervenir.

Los derechos universales son: que cada mujer $u$ hombre, por el simple hecho de serlo, al formar parte de la sociedad en que vive, esta tiene la obligación de proteger la vida, la libertad, la dignidad, la igualdad, la seguridad y la integridad física ${ }^{11}$. A los grupos que con frecuencia y por diferentes causas violan sus derechos se les llaman grupos vulnerados y necesitan una vigilancia por parte del estado. En la lista de grupos vulnerados está la mujer en todas sus etapas de vida, ya sean embarazadas, indígenas, migrantes, personas de la tercera edad, minorías religiosas, con discapacidad o pertenecientes a la población con $\mathrm{VIH}$.

En las instituciones de salud se atiende al $80 \%$ de los grupos vulnerados ${ }^{12}$, lo cual se convierte en un condicionante al atender a dicha población. Recibir los servicios de salud es un derecho social, sobre todo el de protección a la salud, y cuando no se otorga se habla de violencia institucional; para no asumir la institución la responsabilidad se remite a presentarlo como un problema de calidad de atención, reduciéndolo a la relación médico-paciente, aunque la violencia se puede dar en cualquier ámbito institucional, los trabajadores de la salud no son responsables de esta.

Por las condiciones en que desempeña su labor el ginecoobstetra, se desprende la pregunta: ¿qué hacen los especialistas en ginecología y obstetricia que son cuestionados por su actuación durante el proceso de atención del parto en las instituciones de salud pública?

Con el objetivo de agrupar los cuestionamientos que se hacen a los especialistas en ginecología y obstetricia para categorizar los elementos técnicos que determinan su actuación que en ocasiones se constituyen argumentos que determinan que se dio la violencia obstétrica, se pidió a un especialista en ginecología y obstetricia apoyo para conformar aquellas categorías que con frecuencia son objeto de establecer estos cuestionamientos, para poder conceptualizar desde la óptica de la técnica el por qué son acciones tan cuestionadas al momento de intervenir en el proceso de parto.

También los eventos reproductivos son los que están referidos con mayor frecuencia en los artículos especializados en el tema de violencia obstétrica y los que generan mayor controversia, en la población abierta, y se escuchan de manera habitual en el espacio comunicativo. En ocasiones estos temas son en lo que se basan para sustentar una demanda ante la Comisión Nacional de Arbitraje Médico.

Se aislaron dieciséis eventos y se construye un cuadro con las razones técnicas del especialista para realizar las diferentes intervenciones. Para mostrar el contexto y confrontar las opiniones respecto a la atención a la mujer que sirva para hacer este análisis se tomó en cuenta la visión del médico especialista en ginecología-obstetricia, para que pudiera sustentar desde la técnica de los procedimientos que se tienen que realizar durante el proceso del parto (Tabla 1). 
Perinatol Reprod Hum. 2019;33

\section{Tabla 1. Eventos ginecológicos-obstétricos vs. procedimientos médicos}

Evento

Patologización

Medicación

Parto deshumanizado

Posición libremente escogida

Uso de soluciones o suero

Acompañamiento de la paciente por familiar

Atención del parto vertical

El número de hijos

El intervalo en la gestación

Número de embarazos resueltos por cesárea

Sin control prenatal

Resistencia de la paciente a ser revisada

Tactos por varios médicos

Callar a las pacientes en el trabajo de parto

Episiotomía

Exigencia de la atención cuando acude de manera tardía

\section{Procedimiento médico}

El médico realiza un diagnóstico que repercute en un procedimiento, siempre pensando en el mejor resultado perinatal. En caso de no hacer un diagnóstico exacto a un evento dinámico se llama impericia, no violencia

Se indica para inducir el trabajo de parto, ya que la evolución natural es lenta y puede afectar en casos específicos el resultado perinatal, en la NOM-007*. Está establecido el uso de medicamentos (oxitocina, analgesia obstétrica y soluciones)

Elementos por los que se atribuye este calificativo a la atención del personal de salud en las instituciones durante el parto. El parto tradicional es una modalidad derivado de las culturas prehispánicas que incluyen la posición de alumbramiento, la toma de tés e infusiones y el no tener medicina de patente, entre otros

Técnicamente existe la necesidad de revisar a la paciente durante el trabajo de parto cada $30 \mathrm{~min}$, mediante tacto vaginal para corroborar la dilatación, borramiento, frecuencia cardíaca fetal y tensión arterial de la madre. La técnica obstétrica hace necesaria la presentación decúbito dorsal, porque de pie, hincada o caminando no se pueden llevar a cabo estas revisiones; por lo que aumentaría la morbilidad del binomio

La segunda causa de muerte materna es la hemorragia obstétrica, el tener una línea intravenosa lista facilita el tratamiento oportuno de la paciente para evitar su muerte, y en casos específicos se utiliza para reanimar in utero a los fetos. El querer justificar el uso de infusiones o tés para el manejo del trabajo de parto por las parteras se contrapone a la petición de no usar medicamentos

El manejo de áreas blancas, grises y negras es un requisito por la COFEPRIS ${ }^{\dagger}$, se refiere al control de circulación del personal en áreas físicas para evitar la contaminación de los pacientes y disminuir la morbilidad de estos, lo cual es un proceso difícil de cumplir por el propio personal de salud a pesar de estar calificado y preparado en el área de la salud. El ingreso a estas áreas de familiares complica el tratamiento y pone en mayor riesgo a todos los enfermos de la institución, no solo a la embarazada*

La posición es recomendada por la partera, sin acción alguna de médico ginecoobstetra, a menos que se complique el parto. El parto vertical no es posible por la cantidad de médicos contratados en las instituciones y no tener áreas específicas para este evento de estas condiciones

Una de las funciones habituales del médico es informar sobre los efectos de la multiparidad, la cual aumenta el riesgo de sufrir una enfermedad o complicación durante el próximo embarazo y parto (p. ej., hemorragia obstétrica, preeclampsia o distocia). Siempre, de manera arbitraria, responsabilizan al personal de salud por las complicaciones

Al existir un periodo intergenésico corto aumenta el riesgo de hemorragia obstétrica

A partir de la tercera cesárea aumenta el riesgo de placenta baja, acretismo placentario y hemorragia obstétrica. En un número importante de casos en los que se decide la cesárea las pacientes no han acudido a control prenatal, y no se tienen los estudios necesarios para un diagnóstico y prever complicaciones; la paciente solo llega a resolver el embarazo

Es cuando la paciente no acude a revisión o no cumple con los estudios solicitados, lo que aumenta el riesgo en el embarazo y resolución

De manera común se solicita autorización para revisión durante el trabajo de parto 203 veces, cuando la paciente no acepta se trata de convencerla, pues no realizar el procedimiento es un motivo para la negación del servicio, que está tipificado

Los médicos responsables de una unidad de manera habitual son más de 2, ya que se manejan diferentes turnos y las instituciones son hospitales-escuela, está normatizado el procedimiento para los adscritos y los estudiantes

Este es un malentendido, ya que se recomienda no gritar para encaminar la energía en el momento de parto (pujo)

Se realiza solo cuanto se dificulta el nacimiento del polo cefálico, es un recurso que se encuentra tipificado en los manuales de procedimientos

Todas las pacientes y sus familiares creen que su caso es el más urgente a pesar de que lo dejan para el final. En las instituciones de salud locales y nacionales en promedio de asistencia para vigilancia del embarazo es de dos y media ocasiones en promedio. Puede disminuir la probabilidad de un parto de alto riesgo

*Norma Oficial Mexicana NOM-007-SSA2-2016 modificada y publicada en 7 de abril 2016.

${ }^{\dagger}$ COFEPRIS Comisión Federal para la Protección contra Riesgo Sanitario. 


\section{El contexto de la formación y la práctica de la especialidad}

La formación de los especialistas en ginecología y obstetricia está enmarcada por una serie de condicionantes que impiden una formación integral para el desarrollo profesional. Hace 20 años se modificaron los planes y programas de estudio, partiendo de la premisa básica que el modelo educativo por competencias ${ }^{13}$ sería la mejor opción para incrementar el rendimiento académico de los estudiantes; junto con esto se fue abandonando el fundamento filosófico de la medicina, ya que se argumentó que la filosofía no servía y en lugar de esa materia se deberían implementar otras que tuviera una utilidad en la formación. Se fue estructurando un pensamiento pragmático incapaz de plantearse dilemas y formar juicios ante la disyuntiva, que permita funcionar con la certeza de un principio lógico; al hacer de la medicina solo una técnica, niegan la capacidad de pensar, solo es poseer la competencia, y al enfrentarse a casos reales de verdaderos dilemas y de urgencia se quedan sin elementos que les permitan conceptualizar un dilema ético y por tanto una intervención eficaz.

Otro aspecto de la formación es el de la medicina basada en la evidencia ${ }^{14}$, que parte de un error conceptual, visto que el término original se refiere a que es una medicina aún por comprobar, al no someter a evaluación ciertos conceptos e ideas se hace de la medicina una ideología y no la práctica de una ciencia biológica. Algunos de los conocimientos adquiridos bajo esta visión parten de silogismos, y esta por lo general no muestra evidencia sobre la maniobra técnica, se aboca a la prescripción de medicamentos de última generación y la visión se queda corta en la clínica cuando no se tienen los medios para emplear los artefactos tecnológicos de la biomedicina.

Un fenómeno recurrente para los especialistas durante la fase de formación como ginecoobstetras es que se encuentran en un proceso de desgaste profesional' ${ }^{15}$, debido a las condiciones institucionales que impiden un pleno desarrollo profesional, la relación médico-paciente se ve reducida a la mínima expresión y esto hace que se practique una medicina defensiva.

La relación médico-paciente ya no está mediada ${ }^{16}$ por los principios básicos que perduró por años, se ha generado una relación basada en el temor y la desconfianza que impide en algunas ocasiones que la actuación del médico tenga como base la intervención clínica, y le aboca a restringir su participación por temor a una demanda o denuncia sobre sus decisiones.
Los seguros contra demandas del acto médico se han incrementado notablemente por la frecuente amenaza de denuncias, sobre todo a los especialistas ${ }^{17}$. Cuando se interpone un juicio en contra de un médico que trabaja en el sector público, la institución lo maneja como un asunto de particulares dejando al médico solo, e incluso despidiéndolo por ser un «violador de los derecho humanos".

No se puede descartar que hay personas emisoras de violencia dentro del personal de salud, que de forma intencional violentan los derechos de los pacientes y lo harán siempre, pero son casos aislados; el grupo mayoritario del personal de la salud habitualmente no tienen esa intención, aunque sin proponérselo pueden emitir violencia institucional por desconocimiento $u$ omisión, y esto no los exime de responsabilidad porque tiene fácil solución si se siguen los protocolos de actuación médica frente al paciente para protegerlo contra posibles demandas.

En México, los nuevos conocimientos y la tecnología en el campo médico, en particular en la atención obstétrica, en algunas regiones del país, más que en otras, han provocado el surgimiento de conflictos, etiquetaciones y confusiones. Paralelamente un número importante de mujeres buscan la atención del embarazo, parto y puerperio desde la medicina tradicional, y como esta no se practica en las clínicas del sector salud, algunos grupos lo han calificado como ejercicio de violencia obstétrica, más aún por el uso de nuevas tecnologías. Los procedimientos que se realizan en las clínicas del sector van encaminados a la búsqueda de la salud de la paciente partiendo de lo aprendido en las universidades y los hospitales, que con esta práctica se puede garantizar el cuidado de la madre y el recién nacido con procedimientos derivados de la práctica y la experiencia clínica. Si a pesar de esto se consideran válidas las prácticas tradicionales se ejerce la violencia obstétrica desde la institución, al no poner a disposición los recursos de la tecnología. Al ser el parto uno de los eventos que está revestido de una alta carga simbólica, las interpretaciones en torno al hecho puede tener aristas, múltiples interpretaciones y puntos de vista; si no tiene complicaciones se podría atender bajo cualquier modalidad en centros diseñados para tal fin, pero el $56 \%$ de los casos atendidos en el sector salud son embarazos de alto riesgo ${ }^{12}$ : embarazo adolescente, con obesidad, con enfermedades crónicas y degenerativas, diabetes gestacional, infecciones de transmisión sexual, madres añosas y pacientes con pérdida gestacional recurrente. Generalizar la atención a la manera tradicional puede ser el ejercicio de la 
violencia institucional, cuando no se ponen al servicio de la embarazada recursos hospitalarios y tecnológicos que están a su disposición y va contra la conducta ética del personal de salud, pero también contra la vida de las pacientes si se realiza lo que ellas deseen solo por tradición, sin clasificar el riesgo.

La atención al embarazo tradicional puede proporcionarse donde no exista un centro de salud pública, pueden ofrecer esa atención las parteras; no obstante, cuando el parto resulta complicado se recurre a los servicios de salud si la gestante y el producto se hayan comprometidos, y así dejan al personal de salud sin posibilidades de un parto de bajo riesgo. Muchas de las estadísticas de muerte materna debido a las complicaciones del embarazo se han incrementado debido a la atención médica tardía ${ }^{18}$.

La muerte materna, con cifras de alrededor de 529 mil anuales en el mundo, se da en un contexto de países pobres ${ }^{19}$.En México se sabe que el $25 \%$ de las muertes maternas se da en ambientes extrahospitalario en áreas rurales. Las urgencias, con un buen pronóstico, han permitido reducir la frecuencia de la mortalidad materna y neonatal, y son atendidas por especialistas de la ginecología y la obstetricia ${ }^{12}$.

En regiones de mayor pobreza las muertes maternas se explican por factores directos como las hemorragias, y en las zonas urbanas estos factores son la preeclampsia y la diabetes, que son padecimientos que se ven agravados por el embarazo ${ }^{19,20}$. Los planes de tratamiento tienen que partir de un contexto regional donde se ubica el centro de salud y no puede ser de una manera generalizada, ya que la generalización empobrece la actuación. Se puede reconocer que los dos modelos pueden coexistir, sin embargo, al médico especialista en ginecología y obstetricia no se le puede exigir que atienda un parto de la manera tradicional, esa formación no se da dentro de las universidades o los hospitales que forman a los especialistas, y los dispositivos técnicos están diseñados para la atención del parto decúbito dorsal.

\section{Desde la ética}

Para poder caracterizar y hacer un análisis formal de los aspectos técnicos se debe diferenciar la violencia institucional, la obstétrica, el error médico, la negligencia, los procedimientos inadecuados (mala praxis) y la impericia, ya que dependiendo de su origen será la solución. Una constante es que se está calificando como una conducta delictiva algo que vía capacitación se puede resolver, pero dentro de la práctica médica se tiene tipificado como delito, por eso la importancia de despenalizar el acto médico $0^{10-12}$.

El análisis de los términos 0 actos que algunos grupos emplean para conformar la definición de violencia obstétrica se opone a los términos empleado por los especialistas que teórica y técnicamente sustentan el momento para realizarlos, en algunos casos puede pasar que la técnica fracase y no tratarse de violencia. El juicio ético supone la voluntad y la libertad como elemento fundamental para saber y juzgar un acto. Varios de los errores médicos son involuntarios y en muchos casos no existe la libertad de elegir, ya que el evento se resuelve con lo que los profesionales tienen en el momento y según la circunstancia debido a la urgencia.

El pensamiento occidental sustentaba la tesis de que la moral es un atributo de las sociedades humanas, y la ética una reflexión sobre lo bueno, lo justo y lo que debe ser. Singer ${ }^{21}$ refiere que la primera razón de los juicios éticos es orientar la práctica cotidiana. Bajo esta premisa la ética tendrá siempre un referente social, cuando se observa un número elevado de prácticas carentes de ética, las personas hacen de esas prácticas una normalidad en su conducta y no se reflexiona en torno al evento.

Cuando en el espacio público se habla sobre la urgencia de la ética como forma de funcionamiento, entonces también se establece la necesidad de formular códigos, aunque cuando una sociedad intenta mediante reglamentos, códigos y leyes, regular el comportamiento, más problemas tiene. Con la escisión entre las declaraciones oficiales y la vivencia cotidiana la realidad se hace incomprensible, la interpretación se vuelve efímera, lo que se explica para hoy no sirve para mañana, la lógica pierde sentido porque tiene que ser una lógica cambiante de manera vertiginosa, en corto plazo, se tienen que incorporar nuevos elementos para generar nuevas explicaciones que terminan siendo poco explicativas. En forma latente se encuentra la transgresión como una constante y esta no es pasajera, es un componente importante de la conducta humana. La transgresión es poco estudiada por parecer que no existe 0 que un código puede ser suficiente para regular el comportamiento, no obstante, cuando aparece la transgresión se buscará la fisura de la norma para justificar alguna conducta prohibida como justificante de la legalidad, pero en esencia es comportamiento antiético. La trasgresión como componente del comportamiento de las personas puede manifestarse de distintas maneras, ya sea en forma de corrupción, violencia institucional u obstétrica. 
Mediante códigos, normas y reglamentos de una manera generalizada como si las condiciones de centro hospitalario fueran las mismas en la distintas regiones del país se quiere evaluar la actuación médica en las clínicas de primer contacto y los centros especializados de ginecología y obstetricia, lo cual parece ser un exceso, puesto que si los centros tienen recursos económicos y de infraestructura pueden obtener el conocimiento de vanguardia sobre medicamentos, equipo auxiliar de diagnóstico y técnicas actualizadas, mientras que en otros no existen ni gasas; frente a esta se va conformando la violencia institucional y es factible que por extensión aparezca la obstétrica.

La reflexión sobre los propios actos, sobre la propia responsabilidad, no tiene que ser solo en un momento de urgencia, casos difíciles o extremos, sino que tienen que formar parte del profesional, puesto que con la reflexión y la ética como herramienta se pueden modular los actos y hacer posible una convivencia de respeto con los otros y los pacientes.

\section{Discusión}

En el discurso y en todas las relaciones se manejan códigos explícitos e implícitos además de la representación simbólica, el estado de consciencia y el control de impulsos de cada persona, todos estos elementos se hacen presentes cuando se está interactuando, y aún más en la relación médico-paciente, pero no todos los profesionales tienen conocimientos sobre la comunicación. Una forma común de emisión de violencia obstétrica, sin percatarse, debida al desconocimiento, es filtrando su ideología, sea política o religiosa. La existencia de un protocolo de procedimientos para la atención a las pacientes tiene una utilidad práctica para garantizar la imparcialidad, que el personal de salud no actúe conforme a sus valores, emociones y juicio, y esté protegido para no incurrir en errores éticos por desconocimiento.

Emitir juicios o actuar por sentido común fuera de la disciplina es poner en riesgo a la paciente, se está conformando la violencia obstétrica y esta puede incluir regaños, burlas, ironías y negación del tratamiento. Otro aspecto que se ha criticado a los especialistas es que las pacientes son para la enseñanza de la clínica y la práctica de estudiantes sobre diferentes técnicas, aunque todos estos procedimientos están tipificados por la COFEPRIS (Comisión Federal para la Protección contra Riesgos Sanitarios), la actuación va caminando en una línea imaginaria entre una buena praxis y la violencia obstétrica cuando no se cuenta con la supervisión adecuada.

El trato a la paciente debe ser limitado a lo fenomenológico y este trato por parte del personal de salud puede parecer distante, poco humano y poco compasivo. Sin embargo, sí se puede mejorar la comunicación, si se une el lenguaje al tono afectivo dan significado al mensaje, esto es, un trato más humano, más cálido ${ }^{22}$, sin olvidar que en la práctica clínica ${ }^{21}$ la prudencia es la virtud, que implícitamente destacan las normas escritas y no escritas de moralidad para profesionales. Las características de la relación médico-paciente ${ }^{23}$ son asimétricas y de confianza de parte del paciente 0 como mejor se describe esta interrelación. El saber del médico frente al confiar del paciente ${ }^{14}$.

Es necesario reconocer que la violencia institucional u obstétrica se comete por desconocimiento u omisión. Aunque existen profesionales cuyo actuar es claramente ético, la violencia, al ser institucional, se va derivando a la ginecológica y obstétrica como una consecuencia y no como origen.

El criterio clínico, fundamental para tratar a una paciente, debe ajustarse a los protocolos de intervención o los manuales de normas y procedimientos para evitar poner en riesgo a la paciente y al propio médico con las demandas, en caso de que exista una complicación; al conocer los protocolos de intervención se minimizan los actos, al no regirse estos por la voluntad, que ante situaciones críticas puede fallar.

Los profesionales de la salud deben diferenciar con puntualidad la violencia institucional de la obstétrica. Si se elimina o reduce la violencia institucional, esto contribuirá a que la obstétrica se minimice, como una estrategia de convivencia dentro de las instituciones de salud.

En las prácticas de la sociedad, al tener una estructura de pensamiento patriarcal, los estereotipos de poder, violencia y trasgresión se ven como una normalidad y se reproducen en las familias y quedan en la sociedad, al ser introyectados por cada uno se transmiten a la próxima generación, es asimilada por cada persona que constituye el hogar, estableciéndose como una normalidad en la práctica social. Con la interpretación del mundo mediante una estructura de pensamiento patriarcal se puede engendrar la violencia que impacta, influye, en algunos casos determina, la identidad de los hombres y mujeres. Es aquí, en la construcción de la identidad y la mentalidad, donde la violencia como patrón va moldeando la vida, transmitiéndose simbólicamente de una manera sutil y se incorpora como un hecho intrapsíquico ${ }^{23}$ que, a su vez, 
se constituyen en una práctica cotidiana, y va a determinar la forma de interrelación entre los géneros, lo antiético se naturaliza dentro de lo social y lo profesional. Entonces, los códigos éticos tienen que reconocer que las mujeres tienen distintas necesidades y son diferentes a los hombres, pero solo por la biología y no por jerarquía, y partir de la premisa de que en todas las sociedades, especialmente Latinoamérica, coexisten diferentes culturas, ni mejores ni peores sino diferentes; esto puede encaminar a desechar la cultura de la violencia desde lo esencial en toda relación.

Para el desarrollo biológico, psíquico, cognitivo e interrelacional, las personas necesitan de los otros, es a partir de la convivencia con los otros que se aprende a estar en el mundo, de una manera sana o enferma, es lo que nos hace humanos. Aun creciendo y evolucionado al amparo de otro ser, no a todos les parece importante el cuidar del otro, no es solo el desinterés, es la incapacidad en reconocer la importancia del cuidado; algunos lo entienden y lo intentan mediante el control y el sometimiento dando paso a la violencia ${ }^{3}$.

Otro aspecto que no se puede dejar de observar es que desde que se experimenta una visión empresarial de las instituciones de salud se va perdiendo la identidad como médico, al modificar el concepto de paciente por cliente o solicitante de servicios de salud ${ }^{24,25}$, lo que conlleva un fundamento mercadológico, y esta perspectiva no está inspirada en la objetividad y la mayor racionalidad del acto médico ${ }^{26}$.

En términos de modernidad, al querer funcionar con los fundamentos de la medicina basada en la evidencia solo se están acumulando datos sin posibilidad de aplicación, se está aniquilando la experiencia clínica, que en la consulta externa de primer contacto es fundamental. Ya que el $62 \%{ }^{12}$ de la población que se atiende en los servicios públicos de salud de segundo nivel, el resto no tendrá consulta en un centro especializado de tercer nivel. Donde no se cuenta con recursos de la tecnología, el diagnóstico realizado por medio de la experiencia clínica, que durante muchos años ha sido el fundamento de la medicina, ha logrado la disminución de la muerte materna.

Los seres humanos no nacen éticos, pero la ética se puede adquirir practicando los principios frente al otro, es la práctica socia $^{20}$ la que determina si existe una cultura como un referente de los mismos principios puestos en práctica, pues no puede haber una ética individual sin que esté contenida en una ética social que permita que independientemente de que existan códigos de comportamiento se puedan tener dentro de la estructura de comportamiento la censura de sus propios actos que solo es posible cuando se reflexiona.

El argumento fundamental para proponer que la mujer embarazada sufre violencia obstétrica es que en su atención el parto debe ser más humanizado ${ }^{7}$, aunque se olvida que en la actualidad se está viviendo la etapa de mayor expresión de lo humano y no es la mejor de sus versiones, se parte de una concepción que todo lo humano es la expresión de las virtudes y no de los defectos que caracterizan a la especie. Hablar de la ética es hablar de que las cosas que están pasando pueden ser de otra manera ${ }^{21}$. La filosofía, en especial la ética, es para desarrollar un pensamiento reflexivo, es para cultivar las humanidades, sobre todo en el terreno de la atención a la salud, es uno de los factores que puede contribuir de manera importante para que la medicina no pierda su fundamento reflexivo y se convierta en una ideología, pretende regularse a sí misma, su argumento se hace de un discurso sentimental y no un discurso racional, por eso la importancia de generar una ética laica ${ }^{27}$.

\section{Conclusiones}

Entonces, diferenciar si es un problema violencia institucional, obstétrica, de ética o una mala praxis médica determinará el curso de los acontecimientos, aunque hay que reconocer que los referentes colectivos se han perdido, se está viviendo en una época en la que todos los problemas son individuales y no se trata únicamente de una institución de la salud en crisis. Al existir un contexto ético y ser un consenso social para todos se convierte en un deber comportarse éticamente frente a los problemas cotidianos y en la práctica médica.

\section{Agradecimientos}

Los autores expresan su agradecimiento al Dr. José Gerardo Henales Almaraz.

\section{Conflicto de intereses}

Los autores declaran que no existe conflicto de intereses.

\section{Responsabilidades éticas}

Protección de personas y animales. Los autores declaran que para esta investigación no se han realizado experimentos en seres humanos ni en animales. No se realizaron experimentos con animales de ningún tipo. 
Confidencialidad de los datos. Los autores declaran que han seguido los protocolos de su centro de trabajo sobre la publicación de datos de pacientes. Los datos son confidenciales y se cumplen los requisitos de confidencialidad.

Derecho a la privacidad y consentimiento informado. Los autores declaran que en este artículo no aparecen datos de pacientes. Todas las participantes firmaron su consentimiento informado.

\section{Bibliografía}

1. Platón. La República. Obras completas. Ciudad de México: Editorial EMU; 2015.

2. Laurel A. Rutas de la privatización en salud. La jornada [Internet]. 5 ago 2015. Disponible en: http://www.jornada.com.mx/2015/08/05/opinion/a03a1cie.

3. Ley General de Acceso de las Mujeres a una Vida Libre de Violencia [Internet]. México, Diario Oficial de la Federación 2015. Consultado: 17 de diciembre.

4. Castro R, Erviti J. 25 años de investigación sobre violencia obstétrica en México. Rev CONAMED. 2014;19:37-42.

5. Ley de Acceso de las Mujeres a una Vida Libre de Violencia del Distrito Federal. Publicado en la Gaceta del Diario Oficial de la Federación. Publicada en la Gaceta Oficial del Distrito Federal el 29 de enero de 2008. Última reforma publicada en la Gaceta Oficial del Distrito Federal el 26 de noviembre de 2015 [Internet]. México: Instituto de Investigaciones Parlamentarias. Disponible en: http://www.aldf.gob.mx/archivo-fde12a5698a6daa612ł4515f386b1beb.pdf.

6. Morales CF, Carreño MJ, Luque CM, Sánchez BC. La supervisión como detonador de estrés en la práctica médica: recomendaciones para su manejo. Prinatol Reprod Hum. 2010;24:187-93.

7. Pozzio MR. La gineco-obstetricia en México: entre el "parto humanizado" y la violencia obstétrica. Rev Estud Fem. 2016;24:101-17.

8. Prado Murrieta A. Mujer y salud. Miradas en torno al nacimiento. Clivajes. Revista de Ciencias Sociales. 2016;3:79-91.

9. Valdez S, Hidalgo S, Mojarro I, Arenas M. Nueva evidencia a un viejo problema: el abuso de las mujeres en las salas de parto. Rev CONAMED. 2013;18:14-20.
10. Robles E, Peña N, Barriga D, Robles M. ¿Despenalizar los actos médicos? Una aproximación a partir de una perspectiva dual: Medicina y Derecho. Ginecol Obstet Mex. 2014;82:828-38.

11. Constitución Política de los Estados Unidos Mexicanos. Artículo I. Modificación de 10 de febrero 2014.

12. Ruiloba F, González F. Fuentes de conflicto en la práctica de la Ginecología y Obstetricia. En: Comisión Estatal de Arbitraje Médico para el Estado de Aguascalientes. Fuentes de conflicto en la relación médico-paciente. Reflexiones y propuestas. Gobierno del Estado de Aguascalientes; 2014. pp. 69-92.

13. Arista SJ. Bases conceptuales para competencias históricas en secundaria, de acuerdo al plan y programa 2011. Tesis de especialidad en humanidades no publicada. Universidad Pedagógica Nacional. México: Toluca; 2016.

14. Vázquez BE. La no evidencia de la "evidence". A veinte años del inicio de su uso. Ginecol Obstet Mex. 2014;82:816-27.

15. Pintado C, Penagos C, Casas A. Síndrome de desgaste profesional en médicos y percepción de la violencia obstétrica. Ginecol Obstet Mex. 2015;83:173-8.

16. Vázquez BE. Hipócrates está enfermo de incomprensión y fatiga ante la sociedad ¿Cómo curarlo? Ginecol Obstet Mex. 2010;78(10):559-70.

17. Vera CO. Aspectos éticos y legales en el acto médico. Rev Med La Paz. 2013;19:73-82.

18. González PI, Romero PI, Sámano SR, Torres CJ, Sánchez MG, Chávez $\mathrm{CM}$. Muerte materna desde una perspectiva de violencia de género. 2010;24:60-6.

19. Aguirre A. La mortalidad infantil y la mortalidad materna en el siglo XXI. Pap Poblac. 2009;15:75-99.

20. Ramírez AM, Jiménez B M, Boquer HR, Sandoval JL. Caracterización epidemiológica de las muertes maternas en el IMSS Quintanar Roo del año 2009 al 2013. Rev CONAMED. 2016;21:132-5.

21. Singer P. Ética práctica. Cambridge University Press; 1995

22. March CJ. El derecho del paciente a recibir información. Rev CONAMED. 2014;19:183-6.

23. Bedoya $\mathrm{HH}$, Builes CV. El acto médico como ética de la relación. IATREIA. 2009;22:47-54

24. Aguilar FB. Humanidades médicas. Su vigencia para la práctica clínica. Rev Urug Cardiol. 2014;2:169-72.

25. Rojas RS. La representación de servicios de la salud en la televisión mexicana: potenciales consecuencias en las subjetividades en salud. Salud Colectiva. 2016;12:189-201.

26. Ruiz $\mathrm{CH}$. Veinte años de bioética en México: desarrollo y perspectiva de la Comisión Nacional de Bioética. Cirugía y Cirujanos. 2014;82:699-708.

27. González J. Razones ético-filosóficas de una bioética laica [Internet]. Proteo: Diálogos de ética y bioética; 2011 [consultado el 3 de noviembre 2016]. Disponible en: http: www.dialogos.unam.mx/razones\%2filosofica\%20de\%20\%una\%20bioetica\%.laica.pdf. 